\title{
Resultados del tratamiento de rescate con pralatrexate en micosis fungoide y otros linfomas de células $T$
}

Leonardo Enciso, Bonnel Patiño, Mónica Arévalo, Martha Díaz, Humberto Martínez, Paola Spirko, Diana Otero

Instituto Nacional de Cancerología (INC) (Bogotá, D.C.)

Introducción: presentar los resultados del tratamiento en una serie de 10 pacientes con linfoma no Hodgkin de células T (LNHCT) nodal y cutáneo atendidos en nuestra institución. No existen en nuestro conocimiento estudios que analicen los resultados en la práctica rutinaria.

Materiales y métodos: estudio de tipo serie de casos. Pacientes mayores de 18 años, con diagnóstico de LNHCT de cualquier subtipo, tratados con al menos un ciclo de pralatrexate, y se contará con información sobre los resultados del tratamiento y el seguimiento. Todos los análisis fueron realizados en los paquetes estadísticos R (versión 3.4.3) y Stata 15.

Resultados: fueron incluidos 10 pacientes, el 50\% eran hombres. La mediana de edad fue 47,8 años (IQR 17,04). El 50\% tenía diagnóstico de micosis fungoide (MF); los 5 restantes, otros tipos de LNHCT. Todos los pacientes recibieron previamente esquema basado en antraciclinas. El número de líneas previas fue en promedio de 2,8 (SD 1,03). El 30\% de los pacientes desarrolló mucositis en algún ciclo de tratamiento. No se reportaron infecciones por gérmenes oportunistas o episodios de neutropenia febril relacionados. Un paciente (10\%) logró remisión completa, dos pacientes enfermedad estable. Seis pacientes (60\%) progresaron durante el tratamiento. Al momento de la última valoración, cinco (50\%) de los pacientes había iniciado una nueva línea de tratamiento.

Conclusiones: el tratamiento con pralatrexate en nuestros pacientes con linfoma de célula T ofrece bajas tasas de respuesta, las cuales son de corta duración, y aunque tiene aceptable tolerancia, también presenta alto riesgo de complicaciones, principalmente mucositis. 